\title{
Multi-Device Based AR (Augmented Reality) Visualization \& Interaction of Modeling \& Simulation Analysis Results
}

\author{
Dongwoo Seo1, *Myungil Kim1 and Jaesung Kim ${ }^{1}$ \\ ${ }^{1}$ Korea Institute of Science and Technology Information \\ 245, Daehak-ro, Yuseong-gu, Daejeon, Korea, 34141 \\ \{seodongwoo,*mikim,jaesungkim\}@kisti.re.kr
}

\begin{abstract}
AR(Augmented Reality) technologies enhance users' sense of reality and immersion by augmenting additional information (e.g. images, texts, $3 D$ models, etc.) to the actual environment through cameras, making it possible to convey information and conduct tasks more effectively. This paper proposes multi-device based augmented reality visualization \& interaction system to effectively provide $M \& S^{\prime}$ analysis results. It is particularly useful to utilize AR-based $M \& S^{\prime}$ analysis results in various environments (offices and conference rooms) without geographical restrictions. Also, the user can perform the task via increasingly visualizing \& Interacting the M\&S model and the analysis result in RP(Rapid Prototype) as well as the image based marker on the Augmented Reality
\end{abstract}

Keywords: Modeling and Simulation, Augmented Reality, Engineering Analysis

\section{Introduction}

Life cycles of products have increasingly been reduced in recent years as customer requirements have varied and rapidly changed. This makes companies develop and produce products in a short period of time to meet customer demands. In order to achieve this ends, it is important to reduce time and cost of the product that is being developed through verification at the design stage. Following this trend, a new paradigm aiming to design and verify products in a virtual space is emerging. [1-6].

It needs collaboration between many stakeholders involved in the development to verify $M \& S^{\prime}$ analysis results, which are required to design and develop products. Therefore, many stakeholders involved in the development process in various environments (offices, meeting rooms, and workrooms) should be able to more effectively use $M \& S^{\prime}$ analysis results. In addition, there needs to be a support for users to review results more easily as the level of understanding of $M \& S^{\prime}$ analysis results may vary depending on the level of knowledge of such users.

Augmented Reality (AR) technologies enhance users' sense of reality and immersion by augmenting additional information (e.g. images, texts, 3D models, etc.) to the actual environment through cameras, making it possible to convey information and conduct tasks more effectively. Moreover, AR technologies are used in various areas such as virtual product reviews or manufacturing system designs. Due to the development of smart devices, users can use augmented reality more easily with their smart devices. Thus, smart device-based augmented reality can provide $M \& S^{\prime}$ analysis results to users so that they could review and use the results more easily and accurately without geographical restrictions.

This paper proposes multi device based augmented reality visualization \& interaction system to effectively provide $M \& S^{\prime}$ analysis results. It is particularly useful to utilize ARbased $M \& S^{\prime}$ analysis results in various environments (offices and conference rooms) without geographical restrictions. Also, the user can perform the task via increasingly 
visualizing the M\&S model and the analysis result in RP as well as the image based marker on the Augmented Reality

In addition, users not only augment visualize image-based markets in augmented reality, but also augment visualize M\&S models and its analysis results to RP and utilize them to conduct tasks. Particularly, users can not only apply simulation results, but also various materials and use a smart device's touch interface to interact with M\&S models.

This paper is comprised of the following: In Chapter 2, the authors discuss relevant research on AR-based M\&S. In Chapter 3, we describe the system structures of the M\&S presented in this paper. In Chapter 4, we explain the methods for augment visualizing simulation results in an augmented reality environment. In Chapter 5, the implemented systems are explained whilst usability evaluations on proposed systems are conducted. Finally, Chapter 6 concludes the study.

\section{Relevant Studies}

\subsection{Visualization of Augmented Reality-based Simulations}

The advantage of visualizing AR-based simulation results is that it can visualize simulation results in real environment through a device's camera [5]. Some researcher used various sensors to carry out real-time simulations on actual objects and augmented simulation results to actual objects using augmented reality [7]. In the ARVIKA project [12], simulation results such as stress and strength analysis were augmented to the actual vehicle undergoing a crash test so that it can instantly be compared to the actual test results. Augmented simulation results and annotations to a traditional 2D-based design and used a web-based application so that many users can collaborate with each other [6].

\subsection{Visualization of Smart Device-Based Augmented Reality Simulation Results}

Smart device technologies have been used in various areas; they can visualize additional information to reality, making users learn and utilize information more effectively. In particular, researchers have conducted studies on how to augment-visualize simulation results using smart devices. Some researcher used VTK4AR framework to visualize product data in augmented reality [7]. Some Researcher used smart devices to augment-visualize simulations of buildings on the outside and made it possible for many users to participate and cooperate [9]. Some Researcher used smart devices to visualize simulations of machines [13]. The method proposed by Weidlich is made up of serverclient method; server reads data and calculates simulation results whilst client renders and visualizes simulation results. Large-scale realistic design platform [4] project uses supercomputer resources to support general-purpose large-scale interpretation and provides virtual reality as well as augmented reality in the pre and post processing stages of simulations.

\subsection{Interaction Using Smart Devices}

Researchers have also been researching ways to interact with visualized objects. Messe et al. used the touch interfaces of smart devices to move and interact with virtual objects. Chun et al. proposed vision-type interactions using the cameras of smart devices. This is a method of tracing hand gestures or fingers through image processing. Ha et al. used RGB$\mathrm{D}$ camera to trace hand gestures and supported interactions. Unlike previous researches, the augment-visualization of $M \& S$ analysis results in this study supports various environments (e.g. offices, conference rooms, workrooms, etc.) and makes it possible to more realistically review the analysis results by matching the results to the actual objects or RP in augmented reality environment. It also supports other materials in addition to $M \& S^{\prime}$ analysis results, which makes it more useful in reviewing designs and verifying products. 


\section{Design and Implementation of Proposed System}

The Modeling \& Simulation system in this paper is an entry-level structure/ fluid system developed by using open sources so that novices can easily and conveniently use the system. M\&S SW assigns models for interpretation and functional conditions through preprocessor phase and interprets structures and thermal fluid analysis through this. The results conducted can be confirmed by interpretation rendering and chart. The whole processes are structured so that users not familiar with M\&S SW can use the system according to the procedures of the workflow (Figure 1).

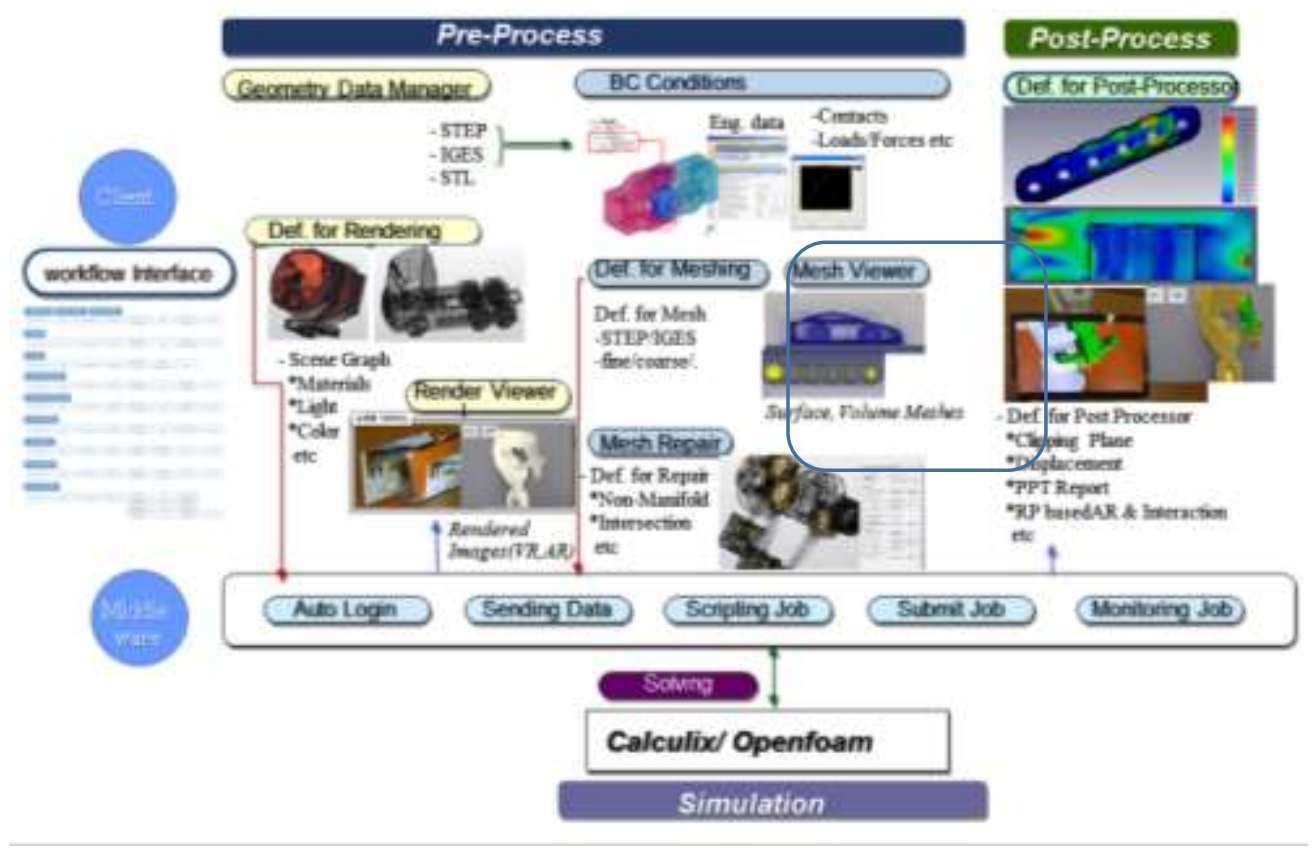

Figure 1. System Concept

It can superimpose augmented reality-based realistic scenes into the $3 \mathrm{D}$ printing physical object in a seamless manner, which can provide more immersive and natural visualization and interaction than conventional virtual reality or augmented reality (Figure 2).

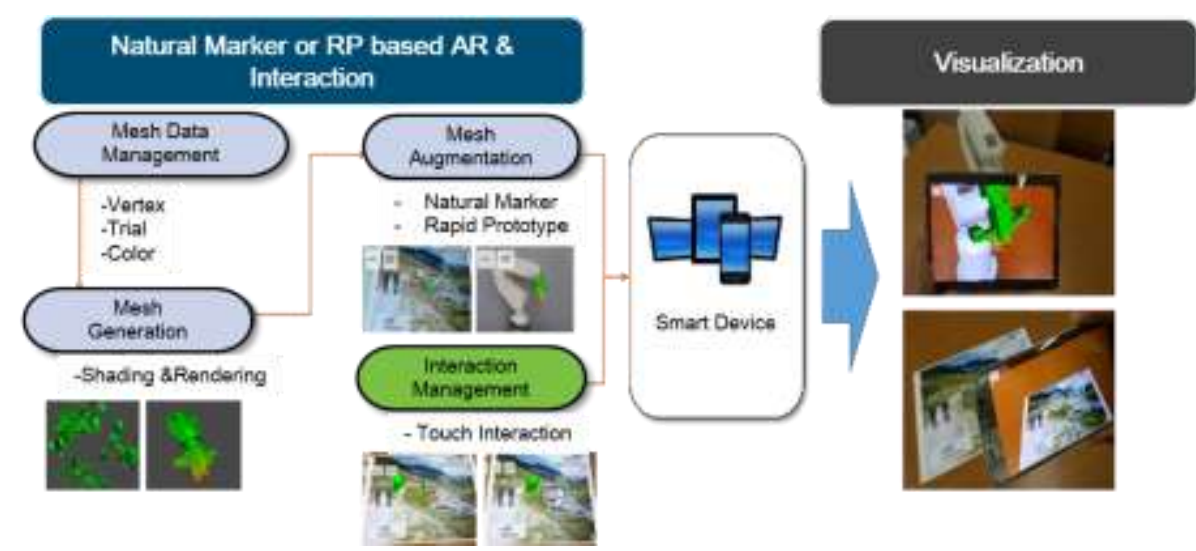

Figure 2. Overall Process

Opencascade, an open source Cad Kernel, was used to develop preprocessing; the kernel reads CAD models, sets a material property for tetrahedron grid model and 
simulation, and assigns functional conditions such as confinement, load, or contact processing.

Calculix, an open source structure analyzer, and Openfoam, an open source fluid analyzer, were used to interpret results, which were installed in parallel to the ezSIM server. Users can access server through authentication information interlocked with $M \& S$ SW and then submit interpretation models to the scheduler. After this, users should independently monitor analyzed information and download the results after completing analysis

The analysis result shows the model by expressing the model by 3-D information on data information, based on file analysis.

\subsection{Integrated Process on Visualization of M\&S Analysis Result}

Augmented Reality on analyzed result shall be treated by the procedure on (Figure, 3). Through formed data, the model shall be modified for RP though shape data, in which elements having problems on shape shall be searched and calibrated. Calibration shape model shall be produced by 3D print to RP. In increasing and visualizing modified shape model, related information shall be used to express the model's shape information, which shall be interoperated and visualized through Unity engine.

Also, through M\&S post-processor, the generated analysis information are extracted, which shall be expressed by the model, and Augmented Reality on final analysis model shall be done.

By using image data processing of M\&S SW's preprocessor, the study generated the STL shape mode, which can be possible to manufacture the RP. For manufacturing the RP model, the study modifies problems on STL model and also, performed grid healing process to increase quality.

As for grid problem search, the study-performed research, centered on Orientation, Flipped Normal / Duplicated Faces // Duplicated Points / Manifold / Folds on Surface / Self-Intersections, etc., in which user provided functions to modify them.

Through such result and by using final data, the RP model was manufactured, which was interoperated by unity data and also, produced integrated treatment for Augmented Reality

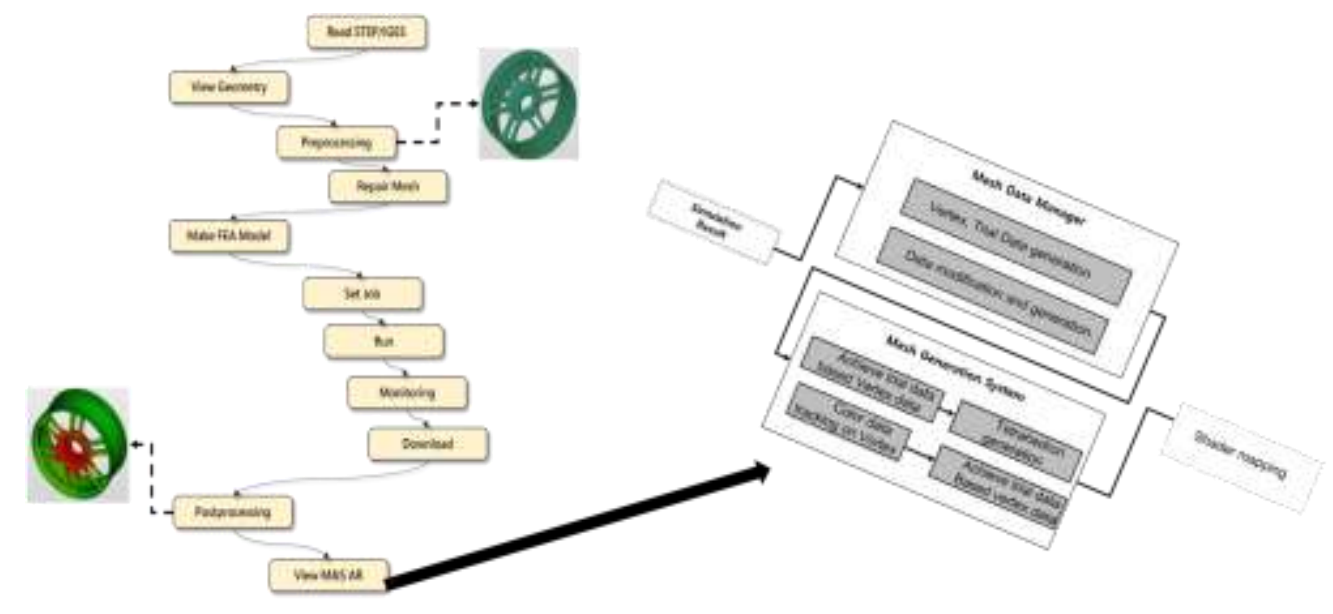

Figure 3. Integrated Process \& Method 


\section{Augmented Reality and Interaction on Simulation Model and Analysis Result}

To perform Augmented Reality on M\&S results by using smart device, the study requested 3 kinds of information, such as vertex, tetrahedron and color. And also, as for each information, the following things could be defined.

Vertex: Location information of point to generate dynamic mesh.

Tetrahedron: Index information of vertex to generate dynamic mesh of trial shape

Color: Vertex's color information to express M\&S result

Based on the three kinds of information, in order to generate dynamic mesh and express analysis result, the study applied Shader on M\&S model and visualized by 3D render. Also, in order to visualize Augmented Reality the study used natural maker or RP to match the dynamic mesh and performed interaction such as moving or rotating by using smart device's touch interface

\subsection{Dynamic Mesh and Shader Configuration to Express Simulation Model's Analysis Result}

For Augmented Reality of M\&S model, based on $M \& S$ results data, in order to express dynamic mesh generation and analysis results, the study configured Shader and applied M\&S model (Figure 4). Just like the above, the study generated dynamic mesh, for which purpose, the vertex, tetrahedron and color were requested.

First, extraction of vertex, tetrahedron and color from M\&S result data shall be gone through. Vertex and color information shall be configured by each line data without specific character string or blank between each line by negative value. Therefore, from $M \& S$ result, vertex, tetrahedron and color shall be abstracted and classified, for which purpose, the data shall be classified according to the number of character string. Also, in order to visualize color information on Augmented Reality, data type shall be converted to RGB. Also, through vertex and tetrahedron, many tetrahedron shall be formed, for which one dynamic mash shall be generated, and also, M\&S model shall be rendered like Figure 7.

The study proposes augmented reality method on $M \& S$ analysis result. As for $M \& S$ analysis result, dynamic mesh was generated and its analysis result applied Shader in order to express analysis result as well as generated Mesh. The study used the smart devices such as smart phone and pad. Also, M\&S model went through augmented reality by using Natural Marker. However, the study did not provide augmented reality on fluid analysis. In addition, as for using RP, Natural Marker should be need because there was not any reason why RP shall be used directly. Therefore, in next study, by putting M\&S result on the fluid analysis on augmented reality and provide it to each user, the study intends to analyze and research fluid analysis-related program, etc. such as OpenFoam [9] and also, by using RP or real materials (actual facilities, etc.) together with smart phone, M\&S model is expected to be visualized more naturally by direct recognition.

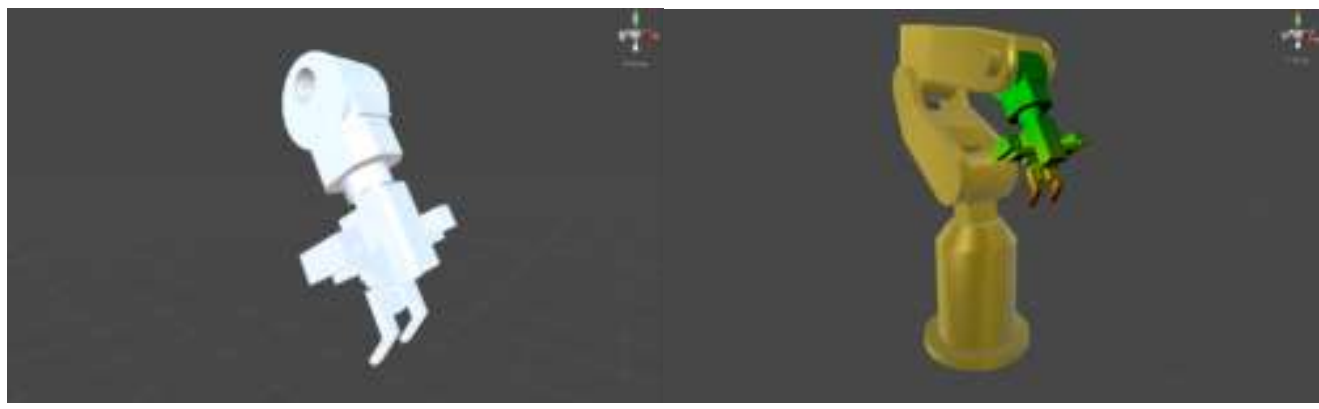

Figure 4. Dynamic Mesh Generation Result 
In order to express M\&S result, Shader shall be applied color information to generated dynamic mesh.

Through Shader, M\&S result can be expressed also, the M\&S model's actual appearance (color, texture, etc.) can be reviewed according to user's needs if needed. In addition, through dynamic mesh generation and Shader application, $M \& S$ model, which is expressed like Figure 8, can be reviewed in the general aspect by additional reality.

\subsection{Augmented Reality Model and Interaction for Simulation Model}

Through dynamic mesh and Shader, rendered M\&S model can applied augmented reality for by interconnecting related $M \& S$ model via $R P$, combined by natural marker or directly by using natural marker. Furthermore, by using smart device's touch interface, in addition to the M\&S model's movement and rotation, interactions, such as changes of color and texture, can be done as well (Figure 5).

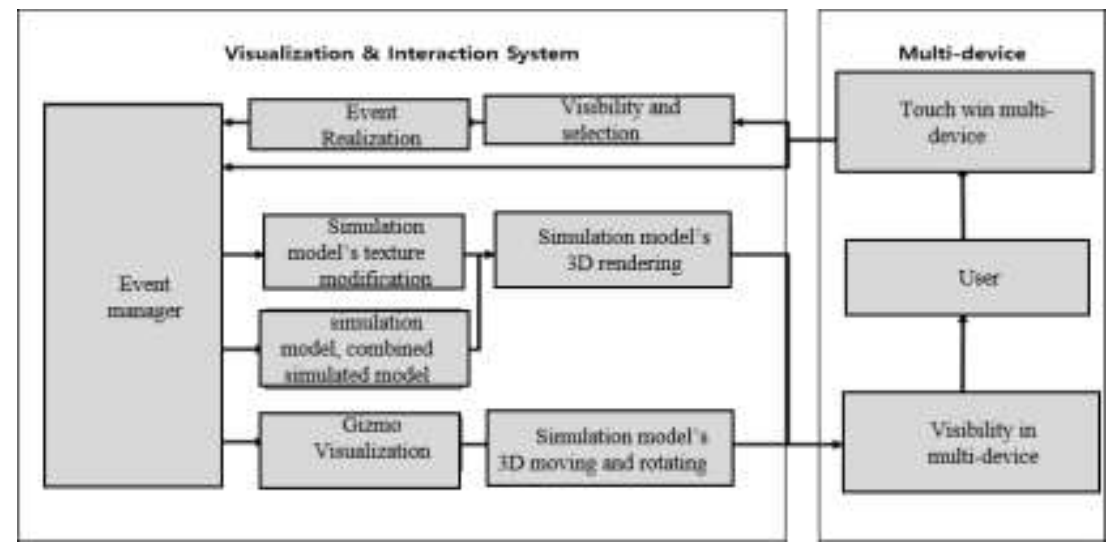

Figure 5. Event Based Visualization \& Interaction Method

In order to increase M\&S model, the study used natural marker. For such fact, from image frame, gained by smart device's camera, the study traced key feature, matched it with natural marker and then, increased camera image of M\&S model. Also, by using RP, combined with the natural marker, study matched the same appearance as M\&S model with actual object, because of which fact the study could express various M\&S model and analysis result. Therefore, high attention and natural image are provided to the user (Figure 6). 

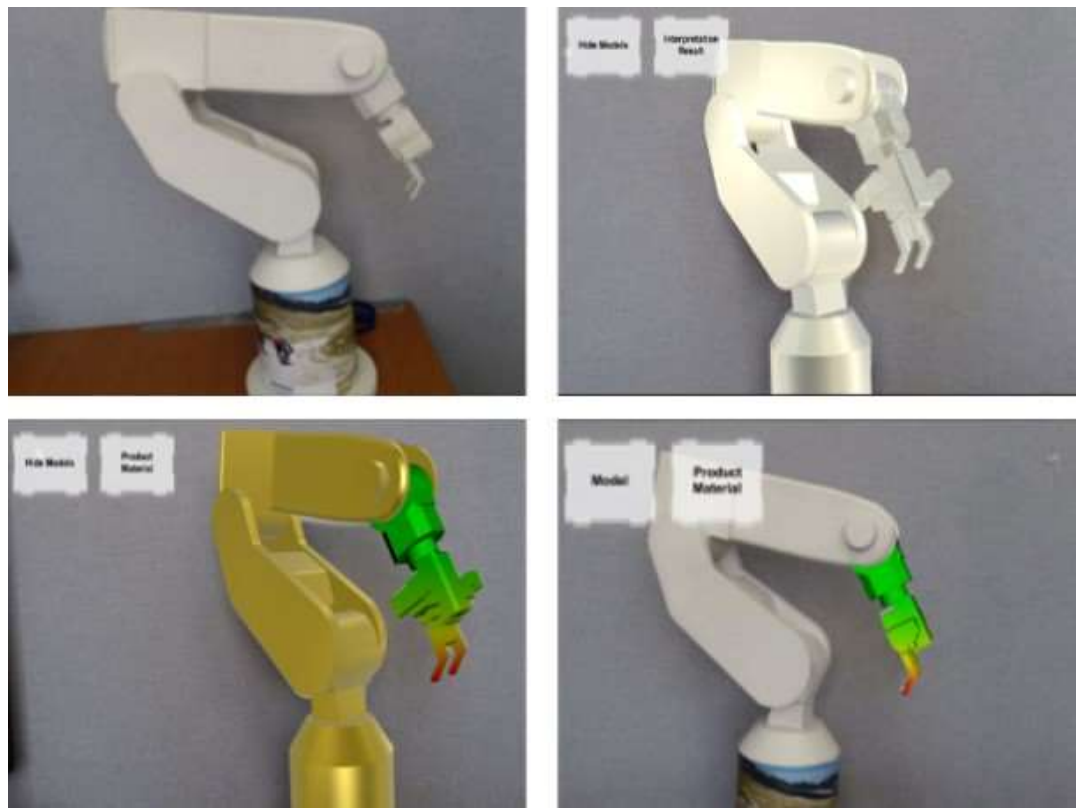

Figure 6. RP Based VR\&AR Visualization

In addition, by using smart device, user can be provided with various environment (office and meeting room, workshop, etc.), based on which fact RP and actual material (actual facility and product) resolved special limits in using RP and actual materials (actual facilities and products), which can be directly used at actual worksite.

Because M\&A aims to verify designed and developed products, mainly, M\&S model shall be under various interaction on M\&S model according to user's needs. For this fact, user can use the smart device's touch interface. Touch interface has been most conventionally used to perform interaction with virtual object, which provides easy and intuitive interaction method. Based on such facts, the study performed moving, rotating, etc. for M\&S model, which can be review in various aspects. In addition, if needed, design review as well as $M \& S$ result verification can be applied.

\section{System Embodiment and Usability Assessment}

By using smart device, under augmented reality environment, for visibility of M\&S model and analysis result, the study used Unity $3 \mathrm{~d}^{\left[{ }^{*]}\right.}$ and Vuforia SDK ${ }^{[*]}$ and also, as the smart device, the study used devices (Lollipop, MarshMellow), based on Android platform operation system (Lollipop, MarshMellow).

The study proposed the smart device, used for qualitative usability assessment on augmented reality method in $M \& S$ analysis results. In addition, in order to analyze usability, the study performed ANOVA analysis.

\subsection{Augmented Reality on M\&S Analysis Results of Cycle Frame (Case 1)}

In this study, the first case is the cycle frame. The cycle frame keeps receiving load while it is running. Therefore, physical damage may happen. Such physical damage may threaten user's safety, etc. so it comes to important problems in frame design and the cost to prevent physical damage has been gradually increased. Thus, as for the cycle frame, the study performed M\&S, used smart device and virtualized augmented reality (Figure 7). The augmented reality on M\&S result of cycle frame uses natural marker. Based on such concept, user can review $M \& S$ result on cycle frame under augmented reality, in which the user can perform interaction through touch interface as well. First by using touch interface, when user selects M\&S model, the Gizmo on moving and rotating is generated, 
at which Gizmo's each axis is selected and also, moving and rotating go through interaction.

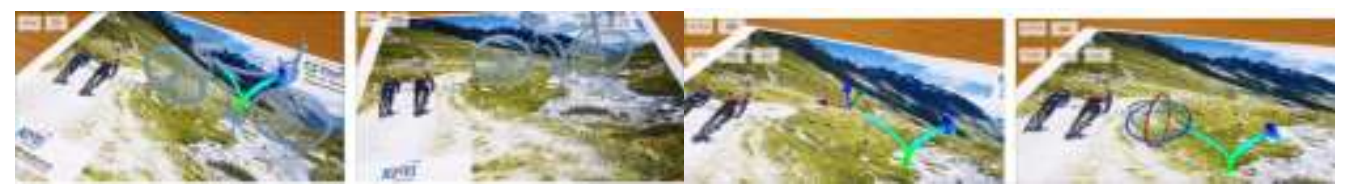

Figure 7. Augmented Reality on M\&S Analysis Results of Cycle Frame

\subsection{M\&S Analysis Result's Augmented Reality on Robot are (Case 2)}

Mainly, robot arm is used for production process. Especially, it is often used for moving product or picking up parts in assembly conveyor. Therefore, the robot arm's end effect is the most important part to develop and design a robot arm.

As for robot arm's end effect, M\&S results' augmented reality was performed by using RP, combined with Natural Marker (Figure 8). RP uses 3D printing, which is manufactured as the same as the robot arm to perform M\&S. Therefore, RP and M\&S models match each other and therefore, according to user's needs, analyzed result and materials come to reviewed together. In addition, beside M\&S model, through applying material on object, on which M\&S is not achieved. Matching between actual RP and M\&S model can be realized. As for M\&S model, the interaction such as moving and rotating does not use the touch interface, instead of which RP is directly moved and rotated.
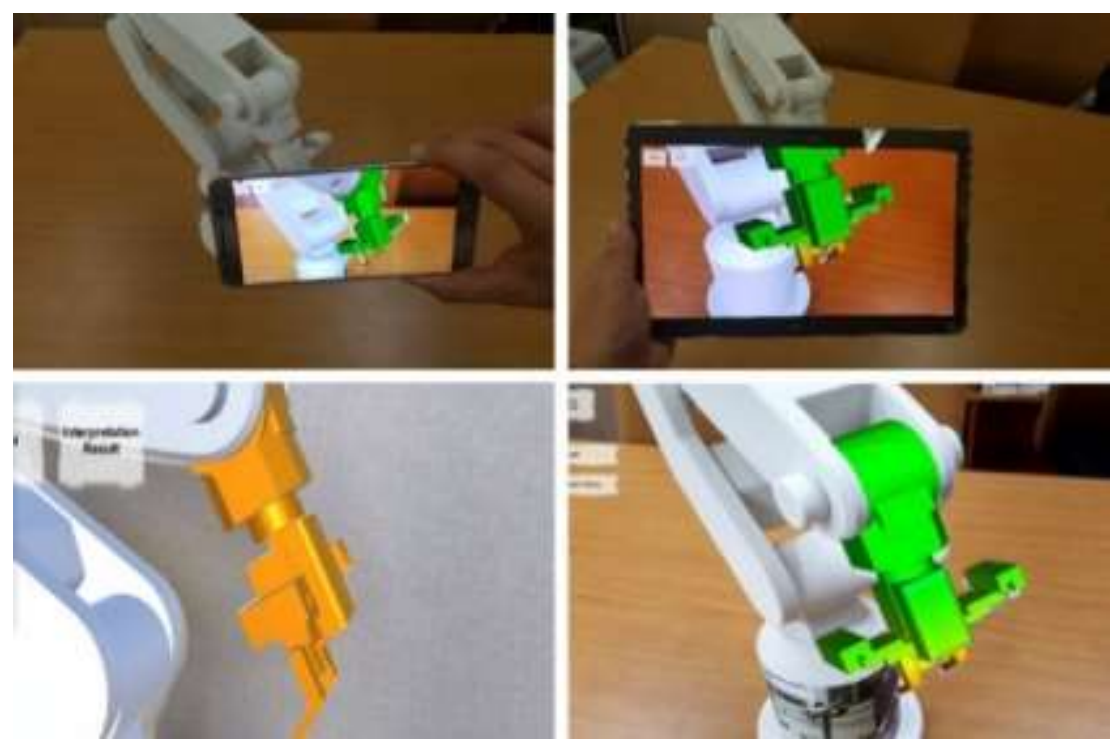

Figure 8. M\&S Analysis Result's Augmented Reality on Robot Arm

\subsection{Usability Assessment's Procedure and Task}

In order to assess augmented reality, eight university students joined the study. Moreover, each joiner used the smart device, which is used routinely. Six students out of eight used one smart device and remained three students used two and more smart devices or used to use them. In addition, two joiners out of 10 used to experience the program used for augmented reality but remained joiners did not experience it, also, who did not experience RP assembly or use as well as augmented reality.

In order to assess the usability, how to visualize $M \& S$ result were large consisted of 
three kinds.

1. AR based Visualization with Cylinder Marker and RP

2. AR based Visualization with Image Marker

3. VR based Visualization

Also, in order to assess such facts, users performed 4 tasks as follows:

1. Only Simulation Model Visualization

2. All Model (Simulation Model + Others) Visualization

3. Simulation Model Material Switching

4. Interaction (Translation + Rotation)

Table 1. Tasks Depend on Visualization \& Interaction Method

\begin{tabular}{|c|c|c|c|}
\hline Task & AR_RP & AR_Image & VR \\
\hline $\begin{array}{c}\text { Only M\&S Model } \\
\text { Visualization }\end{array}$ & $\begin{array}{c}\text { Only M\&S Model / } \\
\text { M\&S Model + Others } \\
\text { with material(No Mask ) }\end{array}$ & $\begin{array}{c}\text { Only M\&S } \\
\text { Model }\end{array}$ & $\begin{array}{c}\text { Only M\&S } \\
\text { Model }\end{array}$ \\
\hline $\begin{array}{c}\text { All Model (M\&S } \\
\text { Model + Others) } \\
\text { Visualization }\end{array}$ & $\begin{array}{c}\text { All Model } \\
\text { Visualization }\end{array}$ & $\begin{array}{c}\text { All Model } \\
\text { Visualization }\end{array}$ & $\begin{array}{c}\text { All Model } \\
\text { Visualization }\end{array}$ \\
\hline $\begin{array}{c}\text { Simulation Model } \\
\text { Material Switching }\end{array}$ & Button & Button & Button \\
\hline $\begin{array}{c}\text { Interaction } \\
\text { (T \& R) }\end{array}$ & Rapid Prototype & $\begin{array}{c}\text { Touch } \\
\text { Interface }\end{array}$ & $\begin{array}{c}\text { Touch } \\
\text { Interface }\end{array}$ \\
\hline
\end{tabular}

According to how visualize, how to perform given task based is following Table 1 . After assessing usability, joiners performed question survey. As given questions, there were 'easy usability', 'interest on interaction', 'easy interoperation', and 'neutrality', which was based on 7 points. Also, in order to analyze it, ANOVA analysis was applied. In addition, in order to analyze such facts, ANOVA analysis was performed. Furthermore, there was request on making priority on 3 kinds of visualization.

\subsection{Result of Usability}

Analysis result on usability was clearly shown like (Figure9) As for 3 kinds of visibility, easy usability $(p<0.05)$ and 'interest on interaction' $(p<0.05)$ showed difference on each other but 'easy interoperation' did not show difference on any others (Figure13).

The result of post analysis on qualitive assessment, The method using AR based RP and other method using AR based Image Market did not show any significant difference. Therefore, there was significant difference between AR based visibility and VR based visibility. 


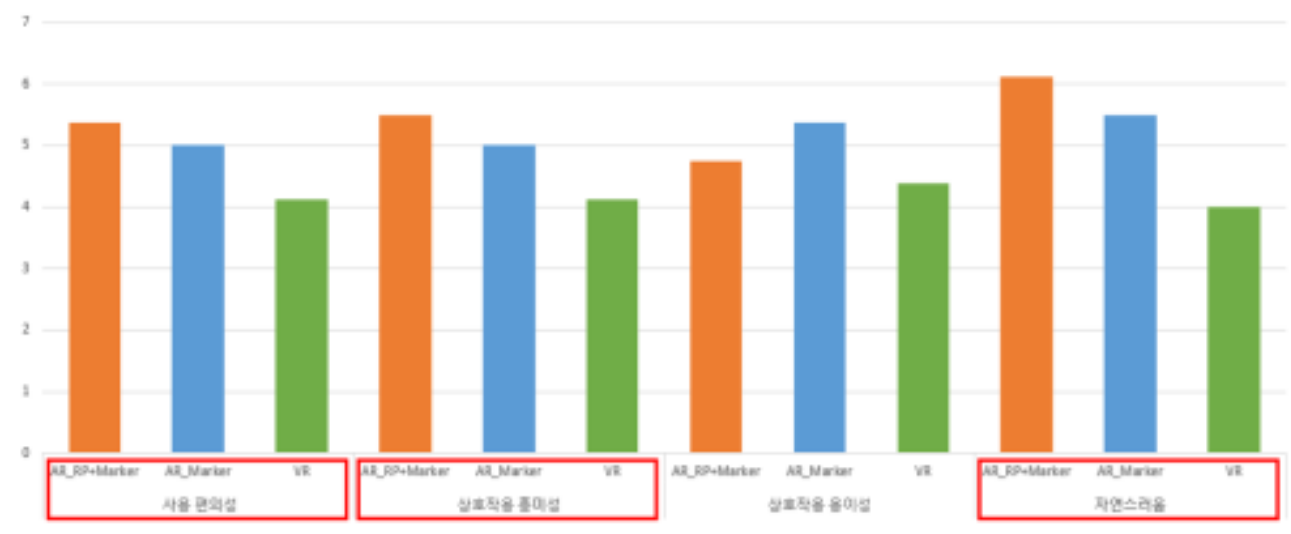

Figure 9. Result of Usability Study

Also, joiner of usability assessment, preference according to the surveyed visibility and its following method was on VR based visibility rather than AR based visibility like (Figure 10)

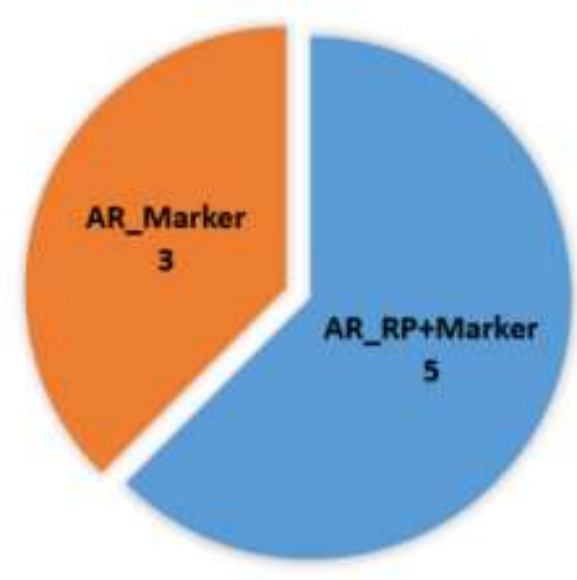

Figure 10. Preference of Visualization \& Interaction

\section{Conclusion}

The study proposes augmented reality method on M\&S analysis result. As for M\&S analysis result, dynamic mesh was generated and its analysis result applied Shader in order to express analysis result as well as generated Mesh.

The study used the smart devices such as smart phone and pad. Also, M\&S model went through augmented reality by using Natural Marker. However, the study did not provide augmented reality on fluid analysis. In addition, as for using RP, Natural Marker should be need because there was not any reason why RP shall be used directly.

Therefore, in next study, by putting $M \& S$ result on the fluid analysis on augmented reality and provide it to each user, the study intends to analyze and research fluid analysis-related program, etc. such as OpenFoam[19] and also, by using RP or real materials (actual facilities, etc.) together with smart phone, $M \& S$ model is expected to be visualized more naturally by direct recognition. 


\section{Acknowledgments}

This study was supported by Supercomputing Modeling \& Simulation Technology Development and Utilization Support (K-17-L04-C03-S03).

\section{References}

[1] R. Suri and R. Hidebrant, "Modeling Flexible Manufacturing System”, Journal of Manufacturing Systems, vol. 3, no. 1, (1997), pp.27-38.

[2] J.H. Kim, C.S. Lee, and S.J. Kim, "High- Performance Domainwise Parallel Direct Solver for LargeScale Structural Analysis", AIAA Journal, vol. 43, no. 30, (2005), pp.662-670.

[3] J.H. Kim and S.W. Woo, "Study on High-Performance Computing Technique for Largescale Structural Analysis", Journal of the Computational Structural Engineering Institute of Korea, vol. 18, no. 1, (2005), pp.37-44.

[4] J.S. Kim, S.M. Lee, J.Y. Lee, H.S. Jeong and S.M. Lee, "Development of Pre- and Post- processing System for Supercomputing based Large-scale Structural Analysis", Transactions of the Society of CAD/CAM Engineers, vol. 17, no. 2, (2012), pp.123-131.

[5] J. Huang, S. Ong and A. Nee, "Real-time Finite Element Structural Analysis in Augmented Reality", Advances in Engineering Software, vol. 87, (2015), pp.43-56.

[6] A.E. Uva, S. Cristiano, M. Fiorentino and G. Monno, "Distributed design review using tangible augmented technical drawings", Comput-Aid Des, vol. 42, no. 5, (2010), pp. 364-72.

[7] F. Bruno, F. Caruso, L. De Napoli, and M. Muzzupappa, "Visualization of industrial engineering data in augmented reality", Journal of visualization, vol. 9, no. 3, (2006), pp. 319-329.

[8] D. W. Seo, J.Y. Lee, S.M. Lee, J.S. Kim and H.W. Park, "Multi-View Supporting VR/AR Visualization System for Supercomputing-based Engineering Analysis Services", Korean Journal of Computational Design and Engineering, vol. 18, no. 6, (2013), pp. 428-438.

[9] V. Heuveline, S. Ritterbusch and S. Ronnas, "Augmented reality for urban simulation visualization", Preprint Series of the Engineering Mathematics and Computing Lab, vol. 16, (2011).

[10] F. Niebling, R. Griesser and U. Woessner, "Using Augmented Reality and Interactive Simulations to Realize Hybrid Prototypes", Proceedings of the 4th International Symposium on Advances in Visual Computing, (2008), pp. 1008-1017.

[11] M. Bhardwaj, "Salinas: A Scalable Software for High-Performance Structural and Solid Mechanics Simulations", Proceedings of Supercomputing Conference 2002, Baltimore, (2002), pp.16-22.

[12] W. Friedrich, "ARVIKA-augmented reality for development, production and service", Proceedings of IEEE/ACM international symposium on mixed and augmented reality, (2002), pp. 3-4.

[13] D. Weidlich, S. Scherer and M. Wabner, "Analyses Using VR/AR Visualization", IEEE computer graphics and applications, vol. 28, no. 5, (2008), pp. 84-86.

[14] A. Mossel, B. Venditti and H. Kaufmann "3DTouch and HOMER-S: intuitive manipulation techniques for one-handed handheld augmented reality", Proceedings of the Virtual Reality International Conference: Laval Virtual, (2013), p. 12.

[15] W.H. Chun and T. Höllerer, "Real-time hand interaction for augmented reality on mobile phones", Proceeding IUI'13, (2013), pp. 307-314.

[16] T. Ha, S. Feiner, and W. Woo, "WeARHand: Head-worn, RGD-D camera-based, bare-hand user interface with visually enhanced depth perception", Proceeding ISMAR'14, (2014).

[17] Unity3d, http://unity3d.com

[18] Vuforia, https://develper.vuforia.com

[19] OpenFoam, http://www.openfoam.com/

\section{Authors}

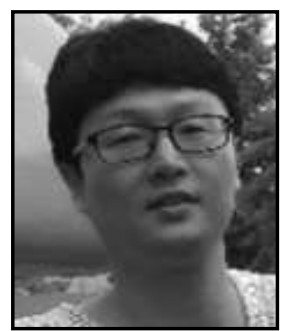

Dong Woo Seo, is a senior researcher at Korea Institute of Science and Technology Information (KISTI), Korea. He received his BS, MS and Ph.D. degrees in Industrial Engineering from Chonnam National University, Korea, in 2003, 2008, and 2012, respectively. His current research interests include AR/VR applications, software architecture, user experience (UX) in CAE service and collaborative design. 


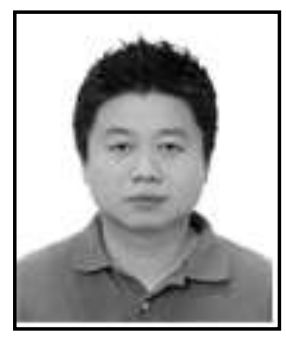

Myungil Kim, is a senior researcher at Korea Institute of Science and Technology Information (KISTI), Korea. He received his BS, MS and Ph.D. degrees in Computer Science \& Engineering from Chungang University, Korea, in 1998, 2000, and 2004, respectively. His current research interests include modeling \& simulation, supercomputing and research network.

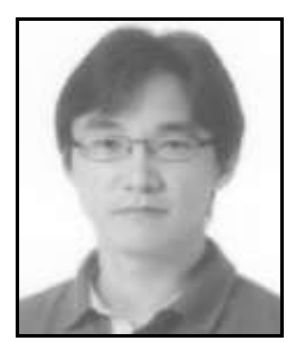

Jae Sung Kim, is a principal researcher at Korea Institute of Science and Technology Information (KISTI), Korea. He received his MS and Ph.D. degrees in Industrial Engineering from Pohang University of Science and Technology (POSTECH), Korea, in 1999, and 2003, respectively. His current research interests include product design, large-scale simulation, VR/AR based post-processing. 